\title{
The effect of mixed convection on the thermal field of horizontal channel flow
}

\author{
Jakub Devera ${ }^{1, *}$, Tomáš Hyhlík ${ }^{2}$ \\ ${ }^{1}$ CTU in Prague, FME, Department of Fluid Dynamics and Thermodynamics, Technická 4, 16607 Prague
}

\begin{abstract}
An experimental study was conducted to investigate the thermal field of low-speed flow of moistair in a horizontal rectangular duct, uniformly heated from below (Poiseuille-Benard flow). Averaged temperature fields were measured in vertical mid-plane using thermocouples. The buoyancy-induced secondary flow is interacting with forced Poiseuille flow which leads to complex flow behaviour with flow reversal along the top surface. Two types of bottom boundary conditions were examined: aluminium plate (only heat transfer) and water film (simultaneous heat and mass transfer) heated to the same temperature. Presence of mass transfer causes stronger transient behaviour above the bottom surface and enhancing of heat transfer, but the overall character of the thermal field remains the same as in case of heat transfer only.
\end{abstract}

\section{Introduction}

Combined free and forced convection can be found in many industrial applications: heat exchangers, chemical vapour deposition reactors and in cooling of electronic components. Mixed convection flow in the horizontal channel is common for these applications. To flow in horizontal channel heated from below is referred as Poiseuille-Benard flow [1].

Bottom heating induces buoyancy driven secondary transverse flow, which alters the velocity field and temperature field. As consequence, heat transfer from the bottom wall is enhanced. Earlier studies were focused on qualifying the heat transfer enhancement and evaluating Nusselt number and its variations along the heated wall $[2,3]$. To understand the nature of the flow and identifying its patterns, flow visualisation studies [4,5] were conducted. Recently, PIV studies were realized to measure the velocity profiles, asymmetric due to the convection from the bottom surface $[6,7]$.

Mixed convection can be characterized by Richardson number [8]

$$
R i=\frac{G r}{R e^{2}},
$$

where $G r$ is Grashof number and $R e$ is Reynolds number. Richardson number characterizes the ratio of buoyancy and inertia forces. If $R i \gg 1$, natural convection is the main convection mode and contribution from forced convection is negligible and vice versa for $R i \ll 1$. If $R i \approx 1$, contribution from buoyancy and inertia forces are in the same relative order of magnitude.

The aim of the study is a visualisation of the thermal field in the horizontal channel subjected to the bottom heating with thermocouples and obtain average temperature field. Two types of bottom isothermal boundary conditions are inspected, aluminium plate and water film, to see whether the simultaneous heat and mass transfer influences the structure of temperature field. Obtained results will be used in upcoming CFD simulation.

\section{Experimental apparatus}

The experimental setup used in the present investigation is shown schematically in fig. 1. At the inlet section of the test rig is a nozzle, which straightens the flow and minimalizes its disturbances. Experiments were conducted in $300 \mathrm{~mm} \times 300 \mathrm{~mm}$ square channel. The test section is $1 \mathrm{~m}$ long and the top and side walls are made from $8 \mathrm{~mm}$ thick plexiglass and insulated by $25 \mathrm{~mm}$ mirelon plates. At the bottom of the test section is water tank (at the bottom and side walls are power regulated heating foils) containing heated water for maintaining stable conditions. An aluminium plate, equipped with 18 temperature sensors (digital thermometers Dallas Ds18b20) to monitor plate's temperature and its uniformity, is dipped in the tank. The maximal deviation of the temperature from the mean value is less than $1{ }^{\circ} \mathrm{C}$. The vertical position of the plate is adjustable, thus it is possible to create water film by lowering the plate. Such arrangement enables investigation of two boundary conditions at the bottom of the test section: heated aluminium plate (heat transfer) and water film (simultaneous heat and mass transfer).

The air mass flow rate through the test section is measured by orifice plate. Differential pressure transducer Setra 265 with range $\pm 125 \mathrm{~Pa}$ is used for measuring the orifice pressure difference. The mass flow rate is evaluated according to standard CSN EN ISO 5176-2.

Inlet and outlet temperature and specific humidity is measured by psychrometers. Each psychrometer consists

\footnotetext{
* Corresponding author: jakub.devera@fs.cvut.cz
} 
of two sheathed RTD probes (PT1000). One of the probes is wrapped by a moisten sock (wet-bulb thermometer). One psychrometer is located at the inlet of the nozzle and two psychrometers are in outlet pipe. Measured outlet specific humidity is used for the calculation of properties of moist air (dynamic viscosity, density) necessary for mass flow rate evaluation. Mass flow rate of dry air is evaluated as

$$
\dot{m}_{\text {dry-air }}=\frac{\dot{m}_{\text {orifice }}}{1+x_{\text {outlet }}},
$$

where $\dot{m}$ is mass flow rate and $x$ is specific humidity.

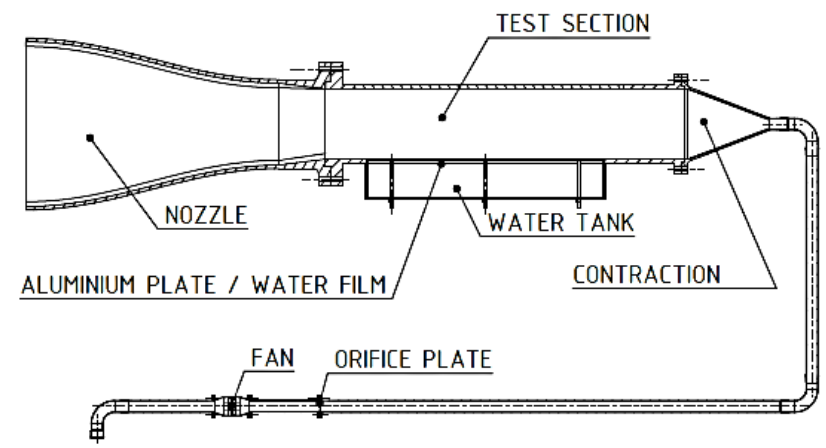

Fig. 1. Schematic of the experimental rig

\subsection{Thermal field measurement}

The thermal field is measured with 10 thermocouples, type K. Data are acquired via NI cDAQ-9174 chassis with four NI 9211 modules which include cold junction compensation. The data are obtained by Matlab (via Instrument control toolbox) and further processed. Thermocouples are mounted to a horizontal traverser unit, vertical distance between probes is $31 \mathrm{~mm}$, the bottom thermocouple is at height of $10 \mathrm{~mm}$ (see fig. 2). Whole $1 \mathrm{~m}$ long test section is traversed by the probe stand, also $0.3 \mathrm{~m}$ in front of and $0.1 \mathrm{~m}$ behind the test section is included, in total 15 horizontal positions (horizontal distance between positions is $100 \mathrm{~mm}$ ). Measurements are conducted in streamwise central vertical plane. In each position is waited for thermal stability, measurement itself in one horizontal position takes 5 minutes. Processing of the data is done in Matlab. Data from each position are averaged

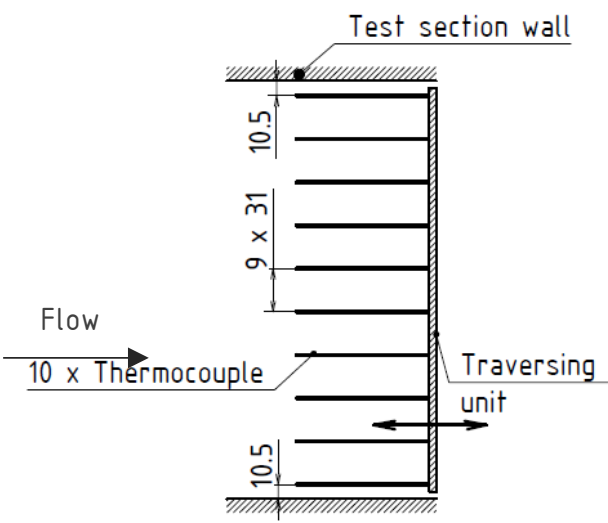

Fig. 2. Horizontal traversing stand with 10 thermocouples used for the thermal filed measurement, horizontal distance between positions was $100 \mathrm{~mm}$ and then interpolated on the measurement grid. Due to the traversing, only averaged temperature field is obtained, any instantaneous behaviour is not captured. The measurement was repeated 3 times in the following days to test the repeatability, the results were comparable.

\subsection{Evaluating dimensionless numbers}

To identify the regime of the flow, it is necessary to evaluate dimensionless criteria from the inlet and boundary conditions, the Reynolds number is calculated according to

$$
R e=\frac{u D_{h}}{v},
$$

where $u$ is the mean velocity in the test section based on the mass flow rate, $v$ is kinematic viscosity evaluated based on inlet conditions, $D_{h}$ is hydraulic diameter. Grashof number is calculated as

$$
G r=\frac{g \Delta T \beta D_{h}^{3}}{v^{2}},
$$

where $\Delta T=T_{\text {boundary }}-T_{\text {in }}$ and $\beta$ is the coefficient of thermal expansion, calculated as $\beta=\left(\frac{T_{\text {in }}+T_{\text {boundary }}}{2}\right)^{-1}$. $T_{\text {boundary }}$ is the temperature of the aluminium plate, water film resp., and $T_{\text {in }}$ is the temperature at the inlet of the experimental rig, $g$ is acceleration due to Earth's gravity.

\section{Results and discussion}

The parameter, which qualifies the relative contribution of forced and mixed convection is Richardson number, $R i=G r / R e^{2}$. Both, Reynolds and Grashof number is evaluated based on hydraulic diameter $D_{h}$. In the present study, the value of Reynolds number is 2045, Grashof number $1.33 \cdot 10^{8}$ and Richardson number is 32 , which implies, that the natural convection is the dominant heat transfer mode. The bottom heating causes buoyancy driven secondary flow, that has a large impact on the thermal field in the channel. Measured thermal field for the vertical mid-plane is shown in fig. 3a for the aluminium plate case (heat transfer) and in $3 \mathrm{~b}$ for the water film case (simultaneous heat and mass transfer). The heated plate, resp. water film, starts at $z=0 \mathrm{~mm}$ and ends at $z=1000 \mathrm{~mm}$. The temperature of the plate, water film resp., is $52.5^{\circ} \mathrm{C}$. The y axis is normalized by hydraulic diameter

$$
y^{*}=\frac{y}{D_{h}},
$$

the thermal field is normalized as

$$
T^{*}=\frac{T-T_{\text {in }}}{T_{\text {boundary }}-T_{\text {in }}} .
$$

Both thermal fields have the same complex structure. Forced Poiseuille flow interacts with buoyancy driven secondary flow. The peak of the cold stream's core is shifted toward the heated plate $\left(y^{*} \approx 0.35\right)$, which caused very steep temperature gradient above the heated plate / 

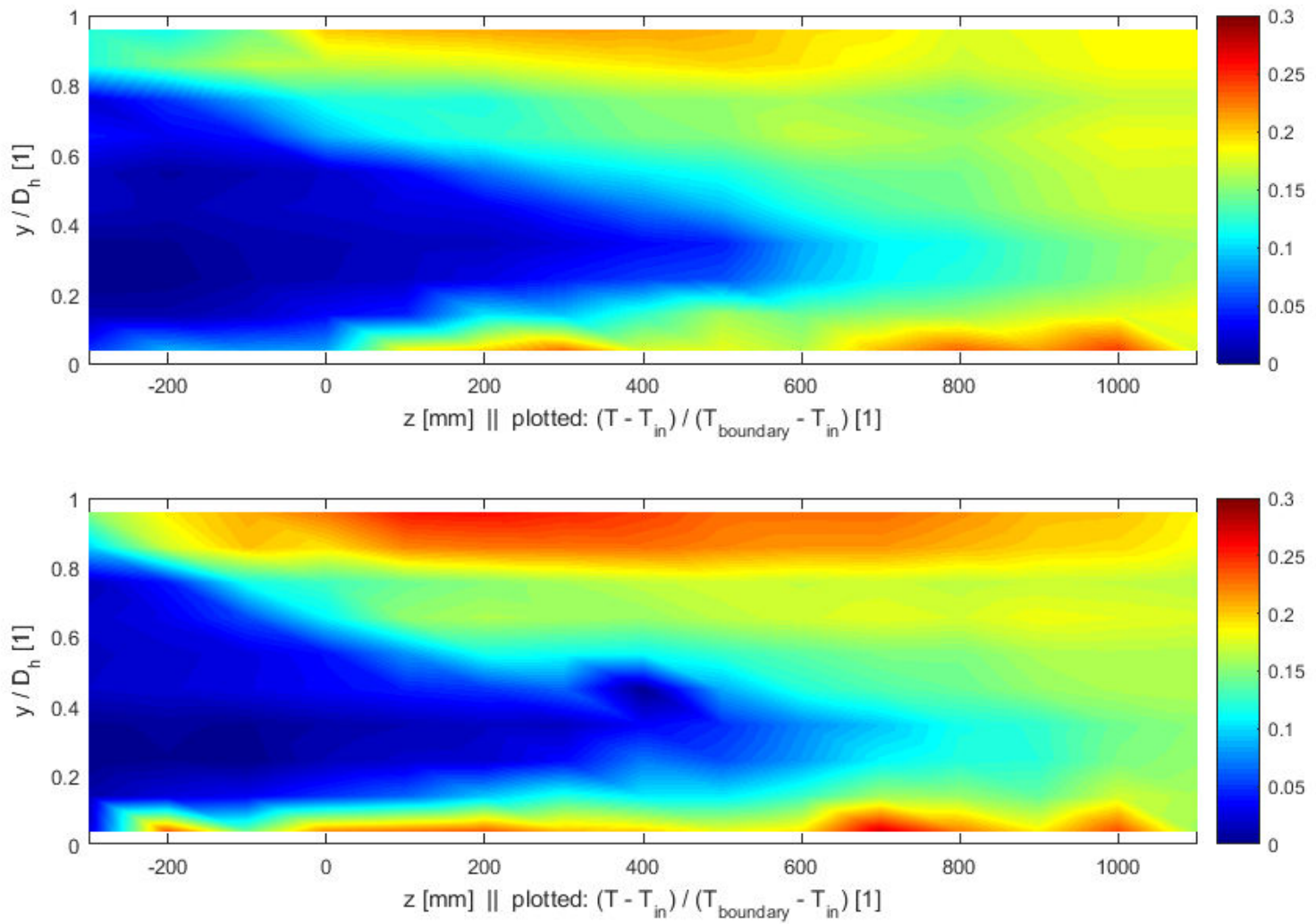

Fig. 3a) (up): mid-plane temperature field above aluminum plate (heat transfer); 3b) (down): mid-plane temperature field above water film (simultaneous heat and mass transfer)

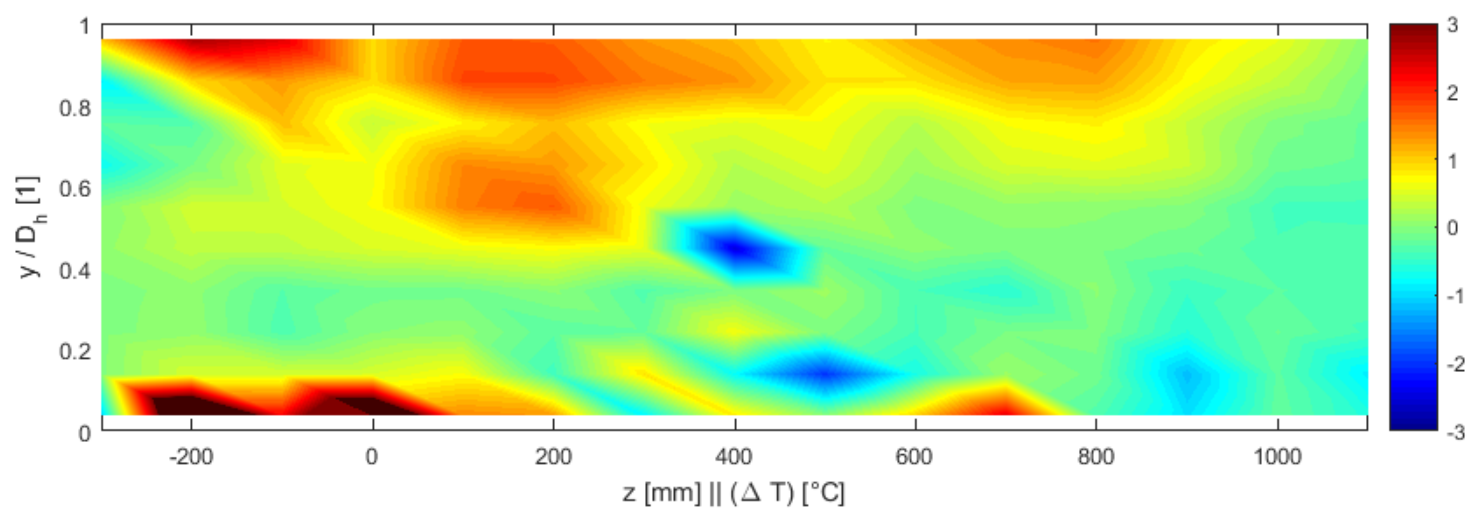

Fig. 4. Difference of temperature fields (nonnormalized); the temperature field above aluminum plate is subtracted from temperature field above water film

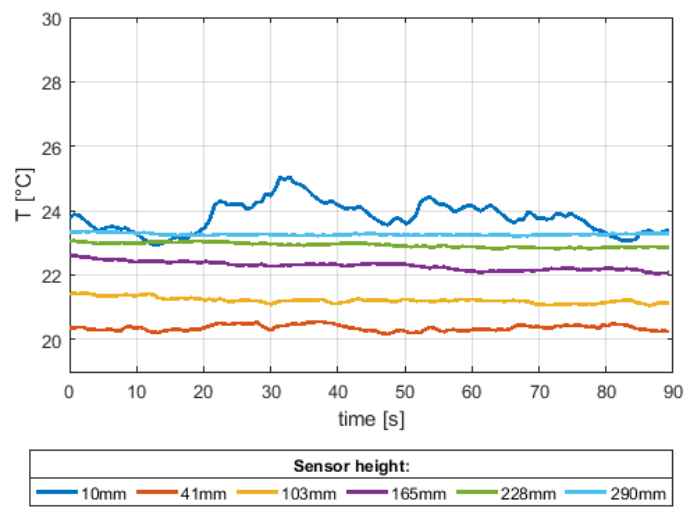

Fig. 5a. Temperature fluctuations vs. time from thermocouples, horizontal position $z=500 \mathrm{~mm}$ for aluminum plate case

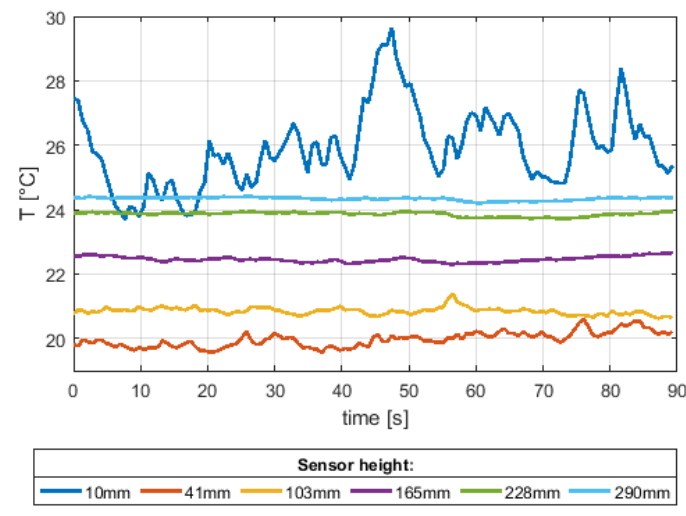

Fig. 5b. Temperature fluctuations vs. time from thermocouples, horizontal position $z=500 \mathrm{~mm}$, water film case 


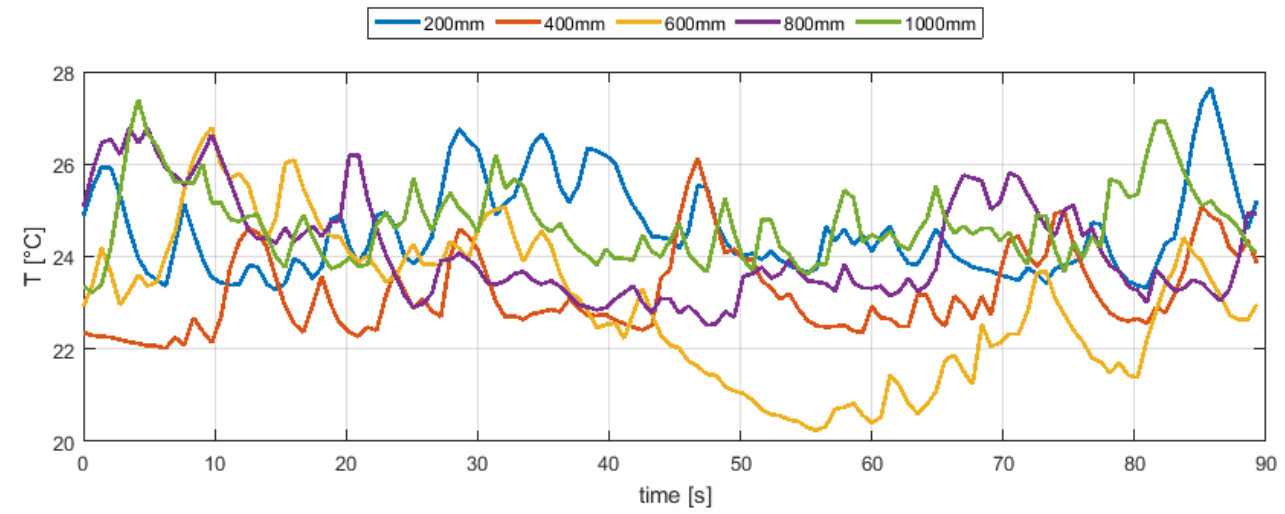

Fig. 6. Temperature fluctuations vs. time from thermocouples, $y=10 \mathrm{~mm}$, different horizontal positions, water film case measurement

hot fluid film. The heated region below the top surface steps into area $x \in(-300,0) \mathrm{mm}$, which is in upwind direction. That is caused by reversed flow along the top surface. The backflow was observed by other researchers studying horizontal mixed convection flow with $R e>10$ $[5-7,9]$. The flow reversal makes the fluid in the upper region hotter than that below due to the convective heat transfer from the downstream fluid [9]. The heated region along the top surfaces covers the entire section and continues to nozzle, thus it not any local behaviour. A large convective cell is created along the entire heated section. The reversed flow a product of complicated 3D flow behaviour because of buoyancy forces, which creates crossflow and positive streamwise pressure gradient [9]. In experiments with water as a working fluid was discovered that reversed flow is induced when $\mathrm{Gr} / \mathrm{Re}^{2}>$ 55 [6]. During this measurement with air, the reverse occurs already when $\mathrm{Gr} / \mathrm{Re}^{2}=32$.

As was mentioned, two boundary conditions were investigated: aluminium plate and water film. Mass transfer doesn't have any effect on the structure of the thermal field, it is the same in both cases, but the thermal field in the second case is hotter in the upper region. The fluid is enhanced by the hot water vapour, which leads to the increase of the temperature. The absolute temperature difference of the both fields is in fig. 4. The airflow is hotter in the upper region $\left(y^{*}>0.6\right)$ by $2^{\circ} \mathrm{C}$. Differences are also at height of $10 \mathrm{~mm}$, but there is problem with steep temperature gradient, where even a small displacement of the probe can cause an error in order of degrees Celsius.

The temperature variations versus time are also inspected. The character of natural convection is unsteady, but the signal from the thermocouples is almost stable in both cases, see fig. 5a and fig. 5b except the signal of the lowest thermocouple, from $10 \mathrm{~mm}$. The temperature at this height is fluctuating, the changes are almost $5^{\circ} \mathrm{C}$ for the water film case, $1.5^{\circ} \mathrm{C}$ for the aluminium plate. At height of $41 \mathrm{~mm}$ above the surface, the signal was much less transient, fluctuations were maximally $0.5^{\circ} \mathrm{C}$ for both cases. The transient behaviour is the same in other horizontal positions, in fig. 6 is plotted dependency of temperature on time from the lowest thermocouple from another 5 horizontal positions.

The temperature signal from $10 \mathrm{~mm}$ height from the water film case was subjected to spectral analysis using Fast-Fourier transformation, but any significant frequency wasn't discovered.

\section{Conclusion}

The thermal field of the Poiseuille-Benard $(R i=32)$ flow was measured in the vertical mid-plane using thermocouples. Natural convection is dominant heat transfer mode and has significant impact on the thermal field. Along the top surface of the test section, reversed flow was observed. In case of water film, as a bottom boundary, the airflow in the upper region was hotter by $2^{\circ} \mathrm{C}$. For this case was also observed strong transient behaviour at height of $10 \mathrm{~mm}$ above the water film, the temperature was fluctuating in range of $5^{\circ} \mathrm{C}$ opposite to the aluminium plate case, where the fluctuations was in range of $1.5^{\circ} \mathrm{C}$.

\section{Acknowledgement}

We gratefully acknowledge the support from Centre of 3D volumetric velocimetry (COLA), reg. no. CZ.2.16/3.1.00/21569, supported by operational programme Prague Competitiveness.

\section{References}

1. Bonnefoi, C., C. Abid, M. Medale and F. Papini, Int. J. Thermal Sciences, 43, 791 (2004)

2. Mahaney, H. V., F. P. Incropera and S. Ramadhyani, Numerical heat transfer, 12, 137 (1987)

3. Maughan, J. R., and F. P. Incropera, Int. J. Heat Mass Transfer, 30, 1307 (1987)

4. Wang L. W., K. H. Hou, I. G. Lu and C. F. Hsu, J. Thermal Energy Generation, Transport, Storage, and Conversion, 9, 257 (1996)

5. K. Toriyama and K. Ichimiya, J. Flow Visualization and Image Processing, 17, 69 (2010)

6. Elatar A. and K. Siddiqui, Int. J. Heat and Fluid Flow, 46, 29 (2014)

7. Elatar A. and K. Siddiqui, Int. J. Thermal Sciences, 90, 351 (2015)

8. F. P. Incropera and T. L. Bergman, Fundamentals of heat and mass transfer, 7th ed, Wiley (2011)

9. Choi D.K. and D.H. Choi, Int. J. Heat Mass Transfer, 37, 1899 (1994) 